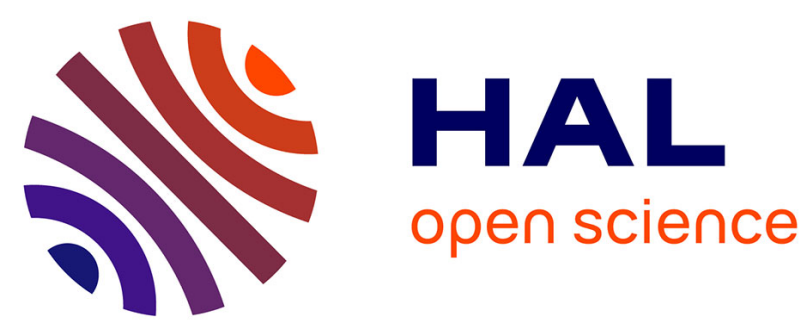

\title{
Correspondence Between Simple 3-D MRI-Based Computer Models and In-Vivo EP Measurements in Swine With Chronic Infarctions
}

Mihaela Pop, Maxime Sermesant, Tommasi Mansi, Eugene Crystal, Sudip Ghate, Jean-Marc Peyrat, Ilan Lashevsky, Beiping Qiang, Elliot Mcveigh, Nicholas Ayache, et al.

\section{To cite this version:}

Mihaela Pop, Maxime Sermesant, Tommasi Mansi, Eugene Crystal, Sudip Ghate, et al.. Correspondence Between Simple 3-D MRI-Based Computer Models and In-Vivo EP Measurements in Swine With Chronic Infarctions. IEEE Transactions on Biomedical Engineering, 2011, 58 (12), pp.34833486. 10.1109/TBME.2011.2168395 . hal-00813802

\section{HAL Id: hal-00813802 https://hal.inria.fr/hal-00813802}

Submitted on 3 May 2013

HAL is a multi-disciplinary open access archive for the deposit and dissemination of scientific research documents, whether they are published or not. The documents may come from teaching and research institutions in France or abroad, or from public or private research centers.
L'archive ouverte pluridisciplinaire HAL, est destinée au dépôt et à la diffusion de documents scientifiques de niveau recherche, publiés ou non, émanant des établissements d'enseignement et de recherche français ou étrangers, des laboratoires publics ou privés. 


\title{
Correspondence between simple 3D MRI-based computer models and in-vivo EP measurements in swine with chronic infarctions
}

\author{
Mihaela Pop, Maxime Sermesant, Tommaso Mansi, Eugene Crystal, Sudip Ghate, Jean-Marc Peyrat, \\ Ilan Lashevsky, Beiping Qiang, Elliot R McVeigh, Nicholas Ayache, Graham A Wright(Member IEEE)
}

\begin{abstract}
The aim of this work was to compare several invivo electrophysiological (EP) characteristics measured in a swine model of chronic infarct, with those predicted by simple 3D MRI-based computer models built from ex-vivo scans (voxel size $\left.<1 \mathrm{~mm}^{3}\right)$. Specifically, we recorded electro-anatomical voltage maps (EAVM) in 6 animals, and ECG waves during induction of arrhythmia in two of these cases. The infarct heterogeneities (dense scar and border zone) and fiber directions were estimated using diffusion weighted DW-MRI. We found good correspondence $(r=0.9)$ between scar areas delineated on the EAVM and MRI maps. For theoretical predictions, we used a simple two-variable macroscopic model and computed the propagation of action potential after application of a train of stimuli, with location and timing replicating the stimulation protocol used in in-vivo EP study. Simulation results are exemplified for two hearts: one with noninducible ventricular tachycardia (VT), and another with a macro-reentrant VT (for the latter, the average predicted VT cycle length was $273 \mathrm{~ms}$, compared to a recorded VT of $250 \mathrm{~ms}$ ).
\end{abstract}

Index Terms - cardiac modelling, MRI, arrhythmia

\section{INTRODUCTION}

$\mathrm{A}$ BNORMAL heart rhythms are often associated with chronic infarct scars and are a major cause $(>85 \%)$ of sudden cardiac death [1]. One dangerous manifestation is ventricular tachycardia (VT), where a "reentry circuit" facilitates a fast re-excitation of the tissue. In such cases, the electrical wave loops fast around relatively large scars and through a so called "isthmus", an abnormal pathway that contains viable myocytes interdigitated within nonconductive collagen [2]. The arrhythmogenic substrate is located at the border zone (BZ) of infarcts and is identified during long and invasive EP studies, which often use surface measurements and aggressive stimulation protocols to induce VT. Should

Manuscript received April 2, 2011. This work was supported by the Canadian Institutes of Health Research under the Grant (MOP93531).

M. Pop, E. Crystal, S. Ghate, I. Lashevsky, B. Qiang and G.A. Wright are with Sunnybrook Research Institute, Toronto, Canada (correspondence: mpop@ sri.utoronto.ca contact phone: 416-4806869; fax416-4805974).

M. Sermesant and N. Ayache are with INRIA Sophia-Antipolis, France.

T. Mansi is with Siemens Corporate Research, Princeton, New Jersey, USA

J-M Peyrat is with Siemens Molecular Research, Oxford, UK.

E.R. McVeigh is with the Department of Biomedical Engineering, Johns Hopkins University, Baltimore, USA. this VT circuit fails to be interrupted, the propagation degenerates into lethal ventricular fibrillation (VF).

To complement the EP study, imaging methods (e.g. MRI) can identify the infarct extent, heterogeneities, and clinical characteristics that predict the patients susceptible to VT/VF [3]. Furthermore, 3D computer modelling is a valuable tool that can be used in cardiac EP to understand the propagation of abnormal excitation waves [4]. Several efforts have been made to develop multi-scale models that integrate imaging, micro-structures and biophysical details [5]. Some researchers have focused on developing such models of small (rabbit) hearts [6], while others on large heart models using noisy ex-vivo fractional anisotropy (FA) from diffusion weighted DW-MR images of canine large hearts [7]. The latter, used a 3D heart model in combination with detailed, ionic equations to study macro-reentrant VT inducibility, but this virtual study lacked any experimental validation. Alternatively, a simple and fast two-variable macroscopic formalism [8] could be used to model spirals [9] and macroreentrant waves $[10,11]$, the latter being associated with macroscopic circuits targeted for ablation therapies $[1,3]$.

In this work, we explore only simple modelling approaches, combined with in-vivo EP measures to detect the extent of scars as well as lead ECG waves (recorded during sinus rhythm and VT) in swine with chronic infarct. Our aim was to compare: i) the scar extent in 3D MRI-model and in electro-anatomic voltage maps (EAVM); and ii) the predictions of (non)inducibility and VT cycle length given by a simple 3D computer model, with the outcome of EP study.

\section{MATERIAL AND METHODS}

\section{A. Electrophysiology Study}

Myocardial infarction was generated in 6 swine by a 90'min by a balloon occlusion of coronary arteries, followed by reperfusion to create heterogeneous infarcts (in accordance to the protocol approved by Sunnybrook Research Institute). Four animals underwent the occlusion of left circumflex (LCX) and two had left anterior descendant artery (LAD) occluded. We demonstrated in another swine study that such heterogeneous areas are realistic, and the evolution of acute infarct (at 2 days) to chronic stages ( $>4$ weeks) is very similar to those in humans [12], as confirmed by histology. 
The in-vivo EP studies were performed at approximately 5 weeks post-occlusion and involved: i) the recording of EAVM on the endo- or epicardium using a CARTO-XP system (Biosense, Webster, CA) normal during sinus rhythm (nSR); and ii) in two swine, the inducibility of VT by following a precise stimulation protocol. The latter employed the application of a train of stimuli S1 that paced the heart fast (to override nSR), followed by several S2-S3 extra stimuli, delivered from the tip of a catheter inserted into the apex of the right ventricle RV (Fig. 1a); this location was also marked in the 3D MRI computer model (see red dot in Fig 1b). Fig. 1c shows an example of EAVM recorded during CARTO acquisition; the infarct is delineated by low voltages (blue-red areas) in bipolar maps. We measured scar areas using the CARTO analysis software, as in routine clinical EP studies [1]. Fig. 1d-e show examples of ECG waves recorded on paper in the two cases where VT induction was performed: one swine had LAD-infarct, and the other one had LCXinfarct. The LCX-infarct demonstrated non-inducible VT after thirteen pacing stimuli $\mathrm{S} 1=550 \mathrm{~ms}$, followed by two $\mathrm{S} 2$ extra stimuli at $400 \mathrm{~ms}$ and one S3 at $300 \mathrm{~ms}$ (thus, S2-S3 coupling interval was $100 \mathrm{~ms}$ ); S3 failed to induce VT, and, consequently, the nSR was recorded again (Fig. 1d). For the LAD-infarct heart, the VT was induced after pacing with eight stimuli $\mathrm{S} 1=800 \mathrm{~ms}$, followed by three $\mathrm{S} 2$ extra stimuli at $300 \mathrm{~ms}$, inducing VT. Several VT cycles were recorded on paper, of approximately $250 \mathrm{~ms}$ each (Fig. 1e). We did not acquire CARTO maps during the two VT inducibility studies.

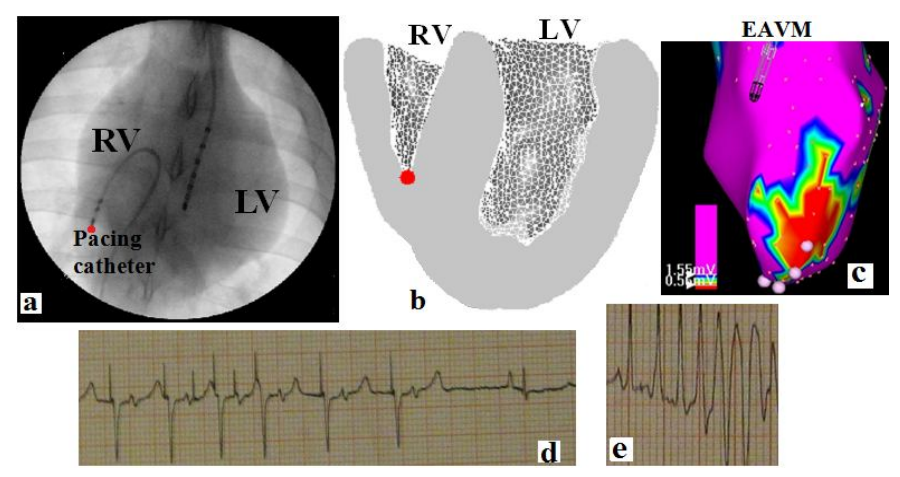

Fig.1. Characteristics of the EP study: (a) fluoroscopic view of the catheters inside the heart during the EP study; (b) the pacing location (red dot) in the 3D model; (c) example of endocardial EAVM (purple indicates healthy tissue); (d) ECG waves obtained in the non-inducible VT case (LCX-infarct); and (e) in the inducible VT case (LAD-infarct).

\section{B. Construction of the 3D MRI-based heart model}

At the completion of in-vivo EP studies, the hearts were explanted, preserved in formalin for few days, and then MRimaged for anatomy, scar characterization and fiber directions using DW-MR. The 3D model was constructed from apparent diffusion coefficient (ADC) maps, which were used to segment the heart into three zones: healthy tissue, BZ and dense scar. Surface meshes were then created from the 3D anatomy MR scans, and volumetric tetrahedral meshes were generated with TetGen package. A function was implemented to measure the scar areas on endo-/or epicardium of the 3D MR model, and to compare them with areas from the EAVM.

\section{Mathematical model}

We used the simple two-variable model developed by Aliev and Panfilov, which is based on reaction-diffusion type of equations [8]. We solve for the action potential using the Finite Element Method, with an explicit Euler time integration scheme [13]. This macroscopic model accounts for heart anisotropy via a diffusion tensor $D$ (which depends on tissue 'bulk' conductivity $d$ ). For instance, the value in the anisotropy ratio is set to 0.14 for a wave propagating almost 2.7 times as fast along the fiber as in the transverse direction.

Some input values for model parameters were taken from our recent optical imaging ex-vivo study [14] in infarcted swine hearts, and they were assigned by zones. The restitution curves found in that study was done by plotting APD90 (ms) vs. cycle length, (CL in $\mathrm{ms}$ ), at different pacing frequencies. For instance in the normal zone, this analysis yielded a measured restitution relation APD90 $=0.218^{*} \mathrm{CL}+151$, and a computed relation APD90 $=0.213 * \mathrm{CL}+160$. These fittings were done using ex-vivo measures, but the AP characteristics at 4-5 weeks are close with those found by others [2] at similar times. The values for $a$ (tuning the duration of AP), $k$ (tuning the up-stroke of AP) and the normalized conductivity $d$ were set as in Table 1. Note that $d$ was set to 0 in the scar. The rest of the parameters ( $\varepsilon$ and $\mu$ ) were set as in [15].

Table 1 Parameters (by zone) in the mathematical model

\begin{tabular}{|c|c|c|c|}
\hline \multirow{2}{*}{ Zone } & \multicolumn{3}{|c|}{ Model parameters } \\
\cline { 2 - 4 } & $a$ & $k$ & $D$ \\
\hline Healthy myocardium & 0.112 & 8 & 3 \\
\hline Border zone (BZ) & 0.2 & 2 & 1 \\
\hline
\end{tabular}

The stimulation in the heart model was achieved as follows: i) the nSR was simulated by applying a $5 \mathrm{~ms}$ duration square maximum amplitude (i.e., $V=1$ since the model output has normalized values for AP), simultaneously on the endocardial surfaces of RV and LV (to mimic activation from Purkinje endocardial terminal buttons); and, ii) the pacing was simulated by applying a train of stimuli $(5 \mathrm{~ms}$ square pulses, with $V=1$ ) replicating the duration and timing as in the EP study, as well as the catheter tip location.

A time step of $5 \times 10^{-5} \mathrm{~s}$ yielded a computational time of 50 min for $1 \mathrm{~s}$ of simulated heart cycle on a regular PC. The mesh for LCX-infarct heart had 212,678 elements, whereas for the LAD-infarct heart the mesh had 245,591 elements (average element size $1 \mathrm{~mm}$, which is sufficient to simulate macro-reentrant VT, as demonstrated in the Appendix).

\section{RESULTS}

\section{A. Comparison between scar area in EAVM and MRI maps}

Figure 2 shows the results obtained after comparing the scar extent measured on the endocardium or epicardium, in the segmented 3D ADC maps and in the EAV maps, respectively. 
The plot shown in Fig. 2 (left) yielded very good correlation $(\mathrm{r}=0.9)$ between the \% scar area delineated in these maps, with EAVM slightly overestimating areas. The Bland-Altman analysis (Fig.2 - right) showed no bias between the methods.
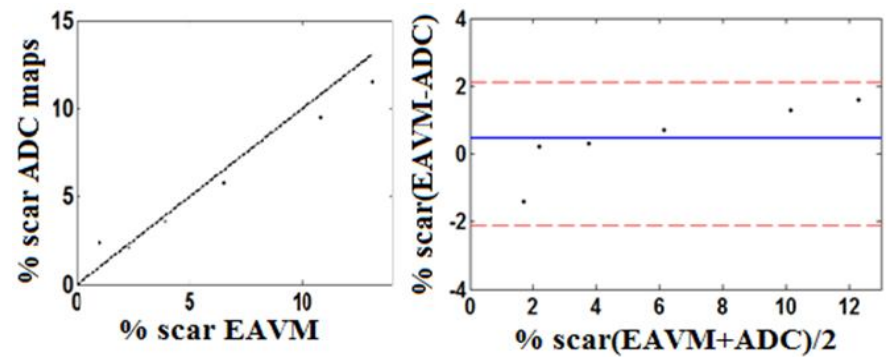

Fig.2. Quantitative analysis for \% area scar delineated by EAVM and ADC-MRI maps: (left) correlation plot; and (right) Bland-Altman plot (blue line corresponds to the mean value, and red line to \pm 2 S.D).

\section{B. Assessment of VT inducibility in the $3 D$ model}

The results shown in Fig. 3 were obtained in the LVX-infarct heart, and illustrate construction and parametrization of the 3D MRI-based computer model, together with an example of theoretical propagation of AP computed for LCX - heart. The left panel shows a 2D axial view through the 3D ADC with elevated ADC values in the scar, the fiber directions from DW-MRI (with severe fibers' disarray in scar), and the 3D $d$ map corresponding to the MRI-based model ( $d$ is set to zero in the scar shown in black, and has reduced values in BZ). The model predicted correctly non-inducible VT for this case.

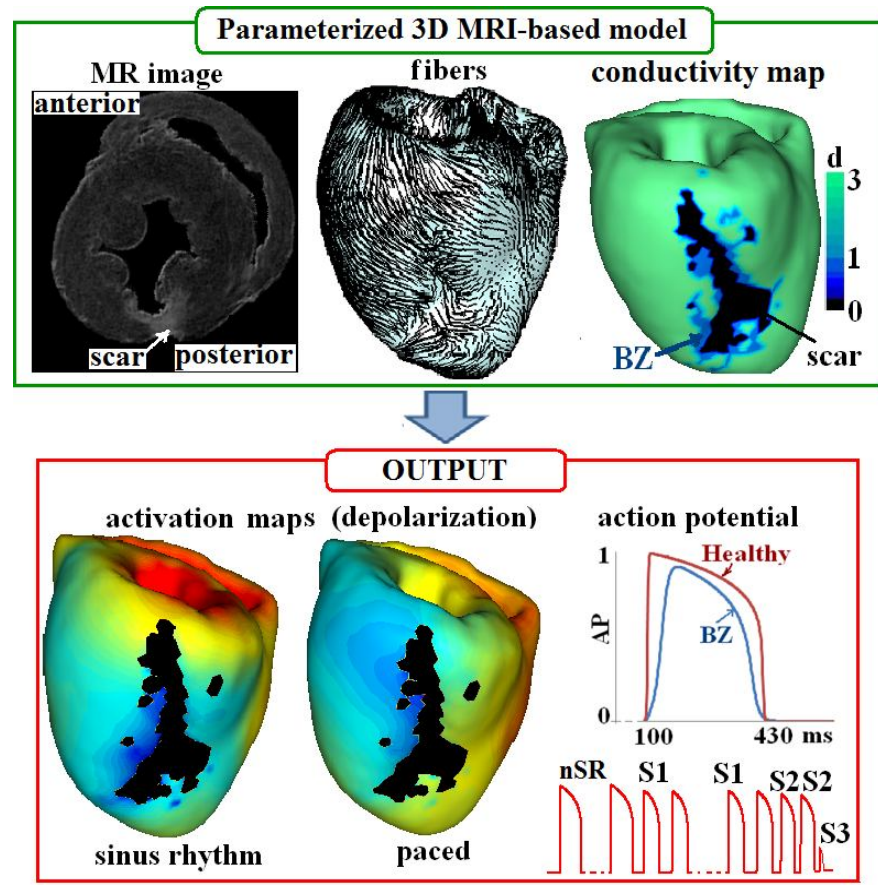

Fig. 3 Upper panel: The 3D MRI-based parameterized model for the LCX-infarct heart (from segmented ADC maps, and fibers from DT-MRI), together with input 3D conductivity map (with scar in black, healthy in green, BZ in light-blue). Lower panel: isochronal maps for depolarization times (color scale corresponding to early activation times in red and late activation times in blue) during nSR and pacing, and computed AP waves before and after the S1-S2-S3 train (resulting in non-inducible VT).
Similarly, Fig. 4 presents results from the 3D model obtained for LAD-infarct. Different geometry of scar and BZ, leads to inducible VT; VT wave looped around scars with a cycle length of $273 \mathrm{~ms}$ (compared to $250 \mathrm{~ms}$ in the EP study).

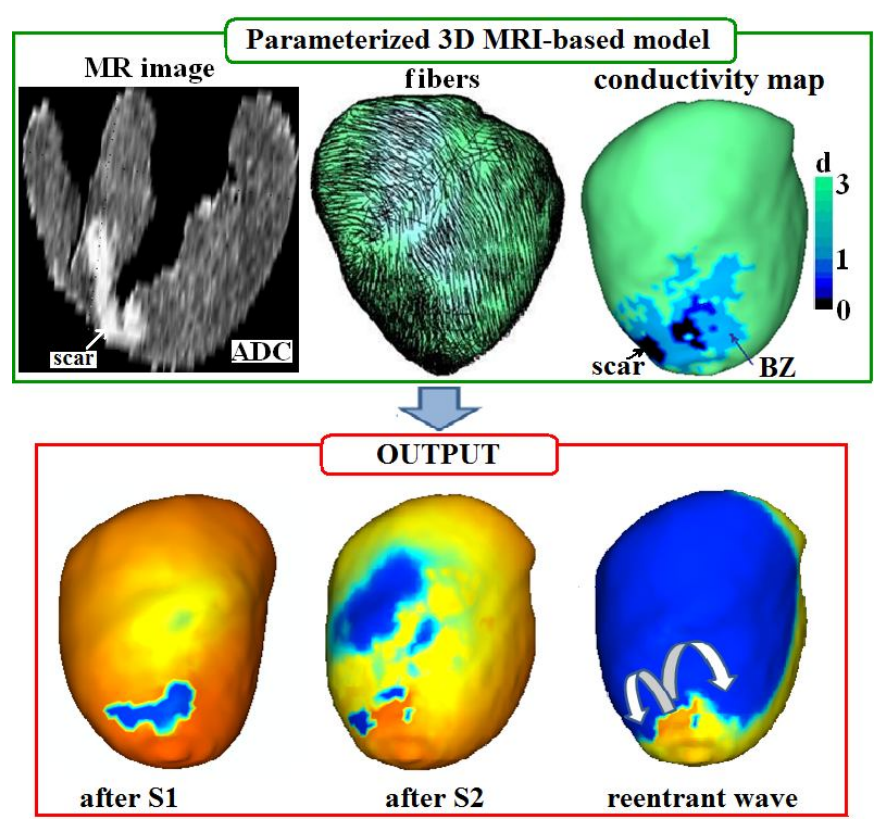

Fig. 4 Upper panel: The 3D MRI-based parameterized model for the LAD-infarct heart from ADC maps, together with a top view through the mesh and the input 3D $d$ - map by zone. Lower panel: simulated isochronal maps after S, S2 and reentrant VT wave propagating around the dense scars (in the directions indicated by the white arrows).

\section{DISCUSSION AND FUTURE WORK}

Prior to integration into clinical applications, 3D MRIbased computer models have to be validated using measures selected to reflect EP phenomena at spatio-temporal scales similar to those considered in simulations [4]. A first step towards this goal is the construction of 3D models from large animal models relevant to human studies, and the comparison between the model output and in-vivo EP measurements.

In this study, we found that ADC maps from DW-MRI identify very well the scar areas in swine hearts with chronic infarcts, and can provide additional information than the surfacic EAVM: transmural extent of scar and BZ. Moreover, the EAVM overestimates the scar area, likely due to their poorer spatial resolution compared to high-resolution MR images. Although we carefully preserved the hearts, small deformation and shrinking in formalin could contribute to the difference between the two methods (in vivo-EP vs. ex-vivo MRI). However, the 3D model was built from ex-vivo scans because DW is not currently suitable to clinical investigations due to motion, image noise, and long scans. Our model is realistic for computational purposes because it integrates the fibres. Another study combining in-vivo EP with ex-vivo contrast-enhanced MR [14], used invasive multi-electrodes that destroyed tissue (hampering DW-MRI) but is superior in that it mapped the depolarization times during VT (from multiple electrode points on the epicardium, simultaneously). 
We acknowledge that the Aliev-Panfilov model is simple compared to ionic models that capture correctly restitution properties and functional VT/VF phenomena like in acute ischemia [4] or in diffuse fibrosis [16] (when spiral waves rotate around very small cores). Our experimental model was developed to reflect characteristics of macroscopic reentries, in the early chronic stage of the infarction (4-5 weeks). This is the time when the scar stabilizes and the patients are suitable to ablation therapies; most of them present with anatomic macro-reentrant VT due to relatively large circuits $[1,3]$. Our simulations were performed for two infarcted hearts: with non-inducible VT and with inducible VT, successfully predicting the outcome as in the in-vivo EP studies. Both cases are clinically relevant, since many patients with prior infarct do not develop VT/VF, and inappropriately undergo implantation of cardioverter devices. For the VTinducible heart, the average predicted VT cycle length was slightly longer (by $23 \mathrm{~ms}$ ) compared to the recorded cycle length, possibly due to input model parameters assigned to different zones after tissue classification. However, we demonstrated in [12] very good correspondence between our classification and the extent of scars in histological images.

We parameterized the model using global values for $k, a$ and $d$ by zone, but these zones could be refined in smaller sub-zones, with different $d$-values. A limitation is that our values were derived from an ex-vivo optical study; thus, further experimentation is needed to assess the in-vivo restitution curve. An in-vivo calibration step should involve endo- or epi-surface mapping under pacing conditions, and computing $d$-maps from depolarization maps; such in-vivo restitution curve could be obtained using monophasic AP mapping catheters [16], and would definitely allow a more precise in-vivo customization of $a$ and $k$.

In summary, we demonstrate good correspondence between several in-vivo EP measures and predictions of macroreentrant VT using a model built from ex-vivo 3D MRI, using a simple mathematical approach. While these results are promising, we next need to refine the experimental model and calibrate the restitution parameters from in-vivo EP paced conditions. Future work will also focus on studying the influence of scar location and geometry on arrhythmia inducibility. With this respect, we will perform more in-vivo VT studies and corresponding modelling, to see when our model fails to predict correctly the outcome of EP study, thus when more complex mathematical models are needed.

\section{Appendix}

We used a thin rectangular slab $(3 \mathrm{~cm} \times 3 \mathrm{~cm} \times 0.5 \mathrm{~cm})$, with $1 \mathrm{~mm}$ element size to determine sufficient space scale. Fig. 5 shows results from a macro-reentry VT that looped around scars through an isthmus of slow conduction. A convergence test for time-steps using following values: $1 \times 10^{-4} \mathrm{~s}, 5 \times 10^{-5} \mathrm{~s}$, $3.5 \times 10^{-5} \mathrm{~s}$ and $2 \times 10^{-5} \mathrm{~s}$. We obtained less than $5 \%$ difference in APD90 values, when using $5 \times 10^{-5} \mathrm{~s}$ compared to $2 \times 10^{-5} \mathrm{~s}$; thus $5 \times 10^{-5} \mathrm{~s}$ seems sufficient for modeling using similar meshes.

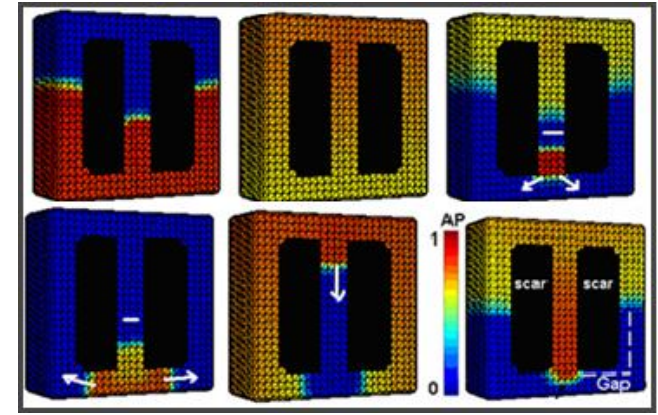

Fig. 5 Macro-reentrant VT in simple rectangular slab: snapshots at different times taken during depolarization phase (red) and recovery (yellow) following S1 and an extra-stimulus S2 (upper row, from left to right). S2induced unidirectional propagation on the isthmus, and generated a VT-wave that looped around the scars (bottom row).

\section{Reference:}

1. Stevenson WG: Ventricular scars and VT tachycardia; Transactionsof American Clinical Association, (2009) 120: 403-12.

2. Ursell PC, Gardner PI, Albala A, Fenoglio J, Wit A: Structural and EP changes in the epicardial border zone of canine myocardial infarcts during healing; Circulation Research, (1985) vol.56, 436-451.

3. Bello D, Fieno DS, Kim RJ et al., Infarct morphology identifies patients with substrate for sustained ventricular tachycardia; Journal of American College of Cardiology, (2005), 45 (7), 1104-1108

4. Clayton RH and Panfilov AV: A guide to modelling cardiac electrical activity in anatomically detailed ventricles (review); Progress in Biophysics \& Molecular Biology, (2008) 96 (1-3) 19-43

5. Bishop M, Plank G, Burton R A B, Schneider J E, Gavaghan DJ, Grau V, Kohl P: Development of an anatomically detailed MRI-derived rabbit ventricular model and assessment of its impact on simulations of electrophysiological function; American Journal of Physiology: Heart and Circulation Physiology (2009), 298: H699-H718

6. Vadakkumpadan F, Rantner L, Tice B, Boyle P, Prassl A, Vigmond E, Plank G, Trayanova N: Image-based models of cardiac structure with applications in arrhythmia and defibrillation studies; Journal of Electrocardiology, (2009) 42 (2) 15

7. Aliev R and Panfilov AV: A simple two variables model of cardiac excitation; Chaos, Soliton and Fractals; (1996a), 7 (3), 293-301

8. Sinha S, Stein K, Christini D: Critical role of inhomogeneities in pacing termination of cardiac reentry; Chaos, (2002), 12, 893-901

9. Pop M, Sermesant M, Peyrat M, Crystal E, Dick A, Wright A: Anatomic reentry: insights from a study in a simple 3D anisotropic wedge model; International Journal of Bioelectromagnetism, (2011) ,11, (1), 133-151

10. Ghugre NR, Ramanan V, Pop M, Yang Y, Barry J, Qiang B, Connelly K, Dick AJ, Wright GA: Quantitative tracking of edema, hemorrhage and microvascular obstruction after acute myocardial infarction by MRI; Magnetic Resonance in Medicine, (2011), in press

11. Sermesant M, Delingette H, Ayache N: An electromechanical model of the heart for image analysis and simulation; IEEE - Transactions on Medical Imaging, (2006), 25, 612-625

12. Pop M, Sermesant M, Liu G, Relan J, Mansi T, Soong A, Truong MV, Fefer P, McVeigh ER, Delingette H, Dick AJ, Ayache N, Wright GA: Construction of 3D MRI-based computer models of pathologic hearts, augmented with histology and optical imaging to characterize the action potential propagation (under revision at Medical Image Analysis, 2010)

13. Nash MP and Panfilov AV: Electromechanical model of the excitable tissue to study reentrant cardiac arrhythmias; Progress in Biophysics and Molecular Biology, (2004), 85, 501-510.

14. Ashikaga H, Sasano T, Dong J, et al, McVeigh ER: MR-based anatomical analysis of scar-related VT: implications for catheter ablation; Circulation Research, (2007), 101, (9), 939-947

15. Ten Tusscher KHWJ and Panfilov AV: Influence of diffuse fibrosis on wave propagation in human ventricule; Europace (2007), 9, 38-45

16. Hao S C, Christini D J, Stein K M, Jordan P N, Iwai S, Bramwell O, Markowitz S M, Mittal S, Lerman B : Effect of beta-adrenergic blockade on dynamic electrical restitution in vivo; American Journal of Physiology, (2004), 287, H390-394 